\title{
Clinical, virological and epidemiological characterization of dengue outbreak in Myanmar, 2015
}

\author{
A. K. KYAW ${ }^{1,2}$, M. M. NGWE TUN ${ }^{1}$, M. L. MOI ${ }^{1}$, T. NABESHIMA ${ }^{1}$, K. T. SOE ${ }^{2}$, \\ S. M. THWE ${ }^{2}$, A. A. MYINT ${ }^{3}$, K. T. T. MAUNG ${ }^{2}$, W. AUNG ${ }^{2}$, D. HAYASAKA ${ }^{1}$, \\ C. C. BUERANO ${ }^{1,4}$, K. Z. THANT ${ }^{2}$ AND K. MORITA ${ }^{1 *}$ \\ ${ }^{1}$ Department of Virology, Institute of Tropical Medicine and Leading Program, Graduate School of Biomedical \\ Science, Nagasaki University, Nagasaki, Japan \\ ${ }^{2}$ Department of Medical Research (Pyin Oo Lwin Branch), Ministry of Health and Sports, Pyin Oo Lwin, Myanmar \\ ${ }^{3}$ Department of Medical Services, Ministry of Health and Sports, Mandalay Children Hospital (550-bedded), \\ Myanmar \\ ${ }^{4}$ Research and Biotechnology, St. Luke's Medical Center, Quezon City, Philippines
}

Received 11 January 2017; Final revision 14 March 2017; Accepted 16 March 2017; first published online 17 April 2017

\section{SUMMARY}

Hospital-based surveillance was conducted at two widely separated regions in Myanmar during the 2015 dengue epidemic. Acute phase serum samples were collected from 332 clinically diagnosed dengue patients during the peak season of dengue cases. Viremia levels were measured by quantitative real-time PCR and plaque assays using Fc $\gamma$ RIIA-expressing and non-Fc $\gamma$ RIIAexpressing BHK cells to specifically determine the infectious virus particles. By serology and molecular techniques, 280/332 (84.3\%) were confirmed as dengue patients. All four serotypes of dengue virus (DENV) were isolated from among 104 laboratory-confirmed patients including two cases infected with two DENV serotypes. High percentage of primary infection was noted among the severe dengue patients. Patients with primary infection or DENV IgM negative demonstrated significantly higher viral loads but there was no significant difference among the severity groups. Viremia levels among dengue patients were notably high for a long period which was assumed to support the spread of the virus by the mosquito vector during epidemic. Phylogenetic analyses of the envelope gene of the epidemic strains revealed close similarity with the strains previously isolated in Myanmar and neighboring countries. DENV-1 dominated the epidemic in 2015 and the serotype (except DENV-3) and genotype distributions were similar in both study sites.

Key words: Clinical, molecular epidemiology, Myanmar, viremia, 2015 dengue outbreak.

\section{INTRODUCTION}

Dengue viruses (DENV) belong to the genus Flavivirus, the members of which are positive-sense single-stranded RNA viruses and can be transmitted

\footnotetext{
* Author for correspondence: K. Morita, MD, PhD, Professor, Department of Virology, Institute of Tropical Medicine, Nagasaki University, 1-12-4 Sakamoto, Nagasaki City, 852-8523, Japan. (Email:moritak@nagasaki-u.ac.jp)
}

to humans by the bite of infected mosquitoes, mainly Aedes aegypti. Infection with DENV can result in asymptomatic manifestation or can cause a wide range of clinical manifestations from mild dengue (DEN) to severe infection [1]. The estimated DENV infections per year is 390 million worldwide and the high incidence makes an economic and social burden not only to developing countries but also to high and middle income countries such as Singapore [2-4]. 
The first DENV outbreak in Myanmar was in 1970 and epidemic outbreaks occur at 2-3 year intervals [5-9]. The number of reported DEN patients has been increasing during the last four decades and the highest peak was in 2015. Moreover, two consecutive outbreaks occurred within the last 3 years (2013-2015) [7]. Virus evolution (genotype or serotype shifting) is an important factor for causing outbreaks [10]. To observe the viral evolution, the molecular study for understanding the circulating DENV serotypes and genotypes during epidemic is essential. Thus, sentinel site surveillance was conducted to understand the molecular epidemiology of DENV at different geographical and environmental areas (central dry zone vs. coastal area) in Myanmar.

Moreover, the previous outbreaks in Myanmar reported that the percentage of primary infection among severe cases were high [7, 8]. We assumed that the high viremia level would be an important factor for causing severe DEN among Myanmar children with primary infection. But, according to literature reviews, the association between the viremia level and disease severity is still controversial and remains an inconclusive issue. Some researchers reported that there is an association between high viremia level and severe DEN disease [11-13] but other reports showed there is no association [14, 15]. Therefore, this study also focused to investigate the association between viremia level and disease severity in Myanmar DEN patients. Thus, to prove the above hypothesis, viral load levels were measured by quantitative real-time PCR method (qRT-PCR) and plaque forming assay using Fc $\gamma$ RIIA-expressing and non-Fc $\gamma$ IIA-expressing BHK-21 cells. In this study, we focused on counting infectious particles by Fcy-expressing BHK cells-based plaque assay because previous studies only focused on counting genome copies by qRT-PCR results which might not provide an accurate picture of the virus infectivity. In addition, characterization of the clinical manifestations and virological patterns of infection among DEN patients and the molecular epidemiology of DENV circulating in Myanmar during the 2015 outbreak could be of help in elucidating our hypothesis.

\section{METHODS}

\section{Study areas}

The areas selected for the study were two dengue epidemic sites in Myanmar: Mandalay and Myeik. Mandalay, an inland city, is located in a central dry zone of Upper Myanmar and close to the border with China, India, and Bangladesh. Myeik, a coastal area in the Mergui archipelago, is located in Lower Myanmar and near the border with Thailand (Supplementary Fig. S1). The mean annual rainfall (central dry zone vs. coastal region: 700 vs. $5500 \mathrm{~mm}$ ) and seasonal temperature generally vary in the two sites (source: Department of Metrology and Hydrology, Ministry of Transport).

\section{Patients and sample collection}

Patients included in the study were those clinically diagnosed to have DEN infection according to WHO 2009 guideline [1]. Laboratory confirmation was done by virus isolation or by detection of NS1 antigen or DENV-specific IgM antibodies in the acute phase serum samples. Severity of the disease was determined by the combined clinical symptoms and laboratory data according to WHO 2009 guideline [1].

Patients were recruited at two hospitals in Mandalay (550-bedded Mandalay Children Hospital and Mandalay General Hospital) and one hospital in Myeik (Myeik Public Hospital). Collection of blood samples was done between July and August during the peak of the 2015 outbreak. Samples were kept at $-80^{\circ} \mathrm{C}$ and all experiments except complete blood count (CBC) were conducted at the Department of Virology, Institute of Tropical Medicine, Nagasaki University, Japan. CBC was done using ABX-Pentra 60 Automated Haematology Analyzer (Horiba Medical, France) in Myanmar.

\section{Serological tests}

In-house DENV-specific IgM capture ELISA and indirect anti-flavivirus IgG ELISA were done on all serum samples following the procedure described previously $[15,16]$. NS1 antigen from serum samples was detected by using Dengue NS1 Rapid Test Kit (InBios, Internationals, Inc, USA) following the instruction of the manufacturer [16]. Patients were confirmed to have primary or secondary infection based on the results of the in-house anti-flavivirus IgG ELISA which was previously validated as a test similar to the WHO recommended Haemagglutination Inhibition test for differentiating primary and secondary DENV infection $[7,8,17]$. Primary infection in clinically diagnosed DEN patients was determined if the acute serum samples were positive for DENV isolation or for 
DENV IgM or NS1 Antigen but with no detectable anti-flavivirus IgG [18]. Secondary infection was determined in patients if the acute or convalescent phase serum samples had an anti-flavivirus IgG antibody titers $>52000$. If patients had an acute phase serum samples only with an IgG titers $\geqslant 3000$ but $<52000$, they were considered to have an undetermined type of infection $[7,8,17]$.

\section{DENV isolation and serotyping}

Serum samples at $10 \mu \mathrm{l}$ volume each were inoculated to Aedes albopictus clone mosquito cell line (C6/36 E2) for DENV isolation [19]. After 7 days of incubation at $28^{\circ} \mathrm{C}$, infected culture fluids (ICF) were harvested and viral RNA was extracted by using Viral RNA Mini kit (QIAGEN, Hilden, Germany). Screening for the presence of DENV was done by Prime Script ${ }^{\mathrm{TM}}$ one-step RT-PCR Kit (Takara Bio Inc., Shiga, Japan) using universal flavivirus primer. One-step RT-PCR method using serotype-specific DENV primers was done to determine the serotypes of the virus isolates [20,21].

\section{Quantification of viremia by using BHK and FcyRIIA-expressing BHK cells}

The viral loads were quantified only from patient serum samples positive for virus isolation.

\section{Plaque assay}

The experiments were performed according to the previous reports with modification [18, 22]. Both Fc $\gamma$ RIIA-expressing BHK-21 and non-Fc $\gamma$ RIIAexpressing BHK-21 cells were prepared in separate 24-well cell culture plates. Each serum sample was diluted 10 -fold from $10^{1}$ to $10^{6}$ with Eagle's minimal essential medium. A $100 \mu$ volume of diluted serum was inoculated to $90-100 \%$ confluent cells in each well and the plates were incubated for $1 \mathrm{~h}$ at $37^{\circ} \mathrm{C}$ incubator with $5 \% \mathrm{CO}_{2}$. A $500 \mu \mathrm{l}$ of maintenance medium including $1 \%$ methylcellulose were then laid over the cells. After 5 days, the plates were fixed with $4 \%$ paraformaldehyde and stained with crystal violet. Plaques were counted and the amount of virus particles in plaque-forming units per $\mathrm{ml}(\mathrm{PFU} / \mathrm{ml})$ was calculated by using the formula: [mean number of plaques per well $\times$ dilution factor]/inoculum volume. In this assay, two independent experimental set-ups were done and the serum samples at different dilutions were inoculated onto cells in duplicates.

\section{$q R T-P C R$}

Viral RNA was directly extracted from patient serum by using the same kit to extract RNA from infected culture fluid. To determine the genome copies of virus, $5 \mu \mathrm{l}$ of RNA was used and amplification of the envelope gene was done by serotype-specific primers using Taq man reagents following the protocol from a previous report and the mean results were reported as genome copies [23].

\section{Gene sequencing and phylogenetic analysis}

The whole envelope protein (E) gene of all the isolated virus strains were amplified by specific primer sets [7]. Sequence was performed by using the BigDye Terminator $3 \cdot 1$ ver and analyzed by ABI Prism ${ }^{\mathrm{TM}}$ Capillary Sequencer 3130-Avant Genetic Analyzer. The nucleotide sequences were aligned by Clustal X, version 2.0 software. With the Maximum Likelihood method using PHYML $3 \cdot 0 \cdot 1$, phylogenetic trees were constructed based on the full $\mathrm{E}$ gene region of the virus strains isolated from this study and from previously isolated strains in Myanmar and its neighboring countries and from the other different regions of the world. The substitution model was selected by jmodeltest- $2 \cdot 1 \cdot 7$ and GTR $+1+\mathrm{G}$ was chosen as the model. Trees were drawn by Fig tree software, version 1.4.2 (FigTree) [22]. The DNA fragments encoding the full length of E protein of DENV were submitted to GenBank (accession numbers from KX357894 to KX357999).

\section{Ethics statement}

The protocol for this study was reviewed by the Ethics Review Committee on Medical Research Involving Human Subjects, Department of Medical Research, Republic of the Union of Myanmar and approved as indicated in the numbered 63/Ethics 2015. Written informed consent was obtained from the adult patients or the parents/guardian of the pediatric patients.

\section{Statistical analysis}

Data analysis was done by using the IBM.SPSS software $20 \cdot 0$ version. Mean values between two groups were compared using unpaired Student's $t$ test. Mean values among groups were compared using oneway analysis of variance (ANOVA) test, and then post-hoc test was performed to detect the significant 
difference among different populations. Non-parametric test was used to compare median values. The $\chi^{2}$ test was done to compare the categorical variables. $P$ value $<0.05$ was assumed as significant in this study.

\section{RESULTS}

Characteristics of the laboratory-confirmed DEN cases

A total of 332 clinically diagnosed DEN patients with single acute phase serum samples was investigated from two study sites in Myanmar. From among them, $280(84 \cdot 3 \%)$ were laboratory confirmed to have DENV infection. Out of the confirmed cases, $47(16 \cdot 8 \%)$ belonged to the category of DEN without warning signs and $184(65 \cdot 7 \%)$ with warning signs while $49(17 \cdot 5 \%)$ were with severe DEN. There were $137(48.9 \%)$ males and $143(51 \cdot 1 \%)$ females. The median age in years for the patients without warning signs was $4 \cdot 0(2 \cdot 3-7 \cdot 5)$ and for the patients presenting with warning signs and severe DEN were $7 \cdot 0(4 \cdot 0$ $10 \cdot 0)$ and $6 \cdot 0(4 \cdot 3-9 \cdot 0)$, respectively. The median age for the patients without warning signs was lower than the other two groups ( $P$ value $0 \cdot 02)$.

Of the DEN-confirmed cases, $228(81 \cdot 4 \%)$ patients were positive for IgM antibody against DENV. Serum samples only from 235 patients were checked for NS1 antigen test due to the limitation of test kit, and 165 showed positive results. Based on the anti-fl avivirus IgG titers, $121(43 \cdot 2 \%)$ cases were classified as primary infection, $111(39 \cdot 6 \%)$ cases as secondary infection and $48(17 \cdot 2 \%)$ cases as undetermined type of infection. For the patients with primary infection, the median age was $6 \cdot 0$ years $(3 \cdot 0-9 \cdot 0)$ and for those with secondary infection was $7 \cdot 0$ years $(5 \cdot 0-10 \cdot 5)$. The distribution of primary and secondary infection in different age groups showed that the commonest age group with DEN cases was $4 \cdot 0-6 \cdot 0$ years (Fig. 1a). Thirty out of 47 patients without warning signs $(63.8 \%)$, and 12 of 49 patients $(24 \cdot 5 \%)$ with severe DEN had primary infection. The number of severe cases with primary infection was quite high. The number of dengue patients with warning signs were highest in all age groups (Fig. 1b).

\section{Clinical and laboratory features of patients with isolated DENV}

At least one of four DENV serotypes was isolated from each of the 104 patients in this study. A comparative analysis on demographic data, laboratory parameters, type of infection, clinical manifestations and infecting serotypes was done among these patients with DENV isolates and was shown in Supplementary Table S1. There was no significant difference in the age, gender, type of infection and WBC (white blood cell count) among the different severity groups of patients. However, platelet counts and hematocrit values were significantly different among the three groups $(P<0 \cdot 05)$. There were also no differences in the clinical manifestations (hematemesis, abdominal pain, liver size) between dengue with warning sign groups and severe dengue (Supplementary Table S1).

\section{Association of serum viremia level and antibody response}

In general, it was noted that the DENV load level of serum samples quantified by using Fcy-bearing cells were higher than by ordinary BHK cells and qRT-PCR method (Fig. 2). Viremia levels were higher for the patients negative for DENV IgM antibody in comparison with those from the patients positive for DENV IgM antibody (Fig. 2a). This finding was consistently significant in all the methods used plaque assay using either type of BHK cells and qRT-PCR. The viremia levels were noticeably high up to day 6 from the onset of fever (Fig. 2b) and the viral load levels were especially consistently high among cases with primary infection up to day 5 of infection (Fig. 2c). The virus titers were also compared between cases with primary and secondary infection and the virus titers were found significantly higher in patients with primary infection $(P<0.05)$ (Fig. $2 d)$.

\section{Comparison of viremia levels among patients exhibiting different levels of severity of DENV infection}

The mean viral loads among the three groups of patients grouped according to the levels of disease severity did not differ significantly $(P>0.05)$ with respect to day of fever (Fig. 3) or to specific serotype (DENV-1, -2, -4) excluding the two cases of mixed infection (Table 1).

\section{Distribution of DENV isolates in the two study areas}

A total of 106 virus strains were isolated from 104 DEN patients with 66 viral strains from patients in Mandalay and 40 from patients in Myeik. In 
(a)

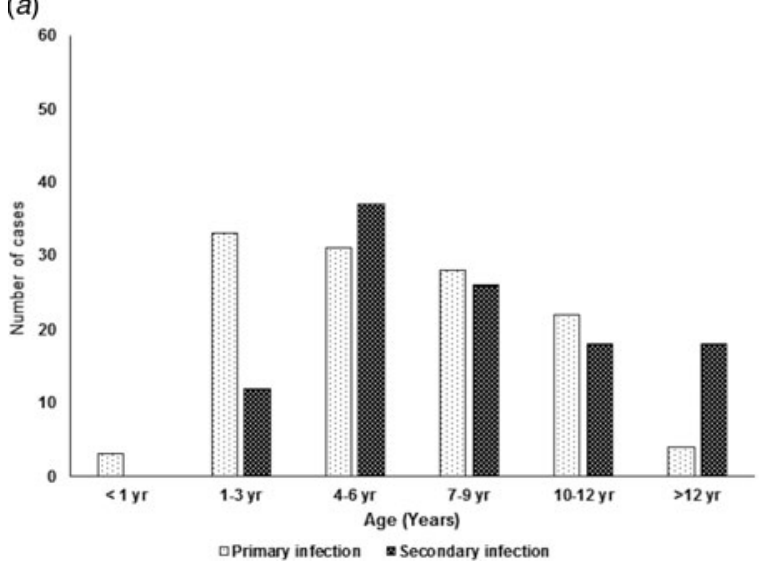

(b)

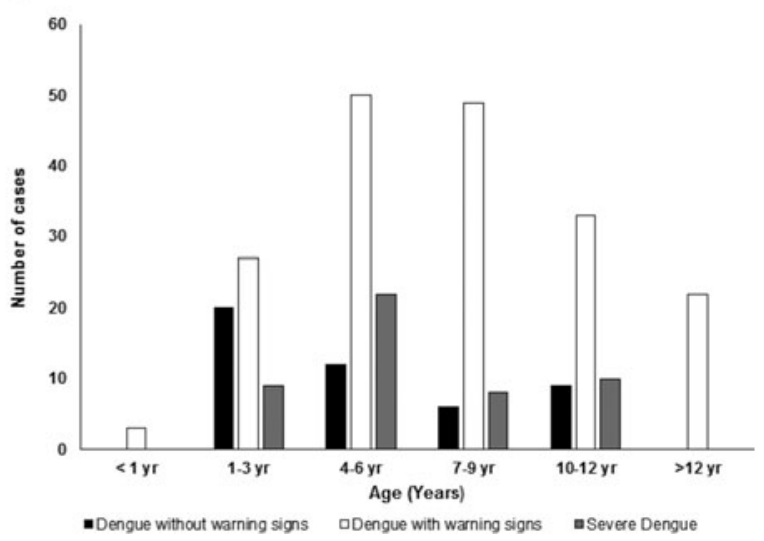

Fig. 1. Distribution of the occurrence of $(a)$ primary and secondary infection, and $(b)$ disease severity at different levels among patients of different age groups.

(a)

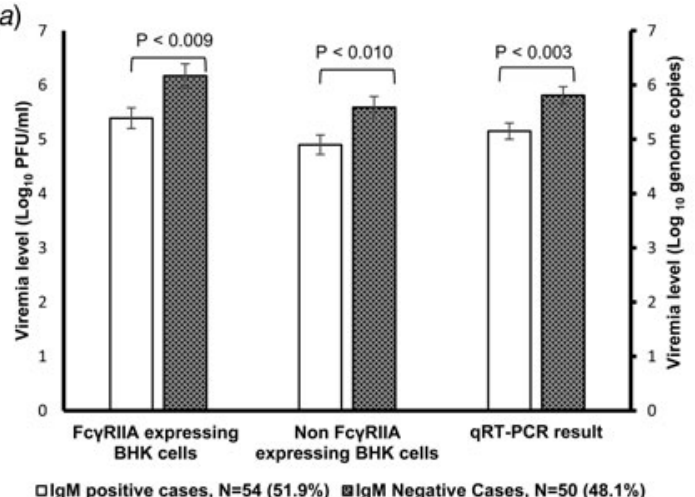

(c)

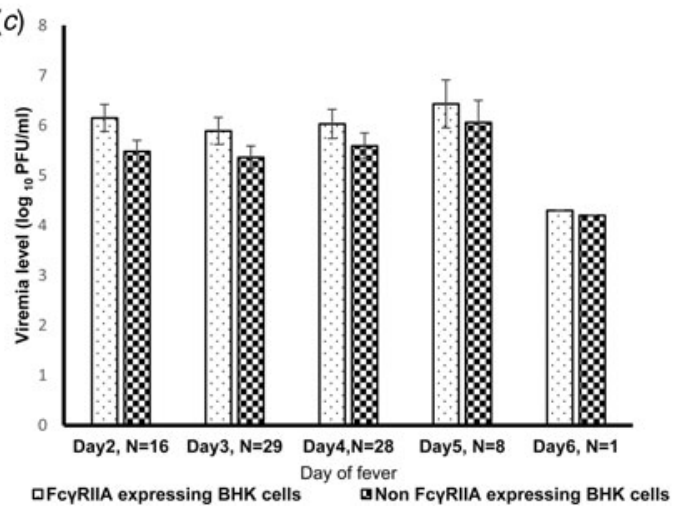

(b)

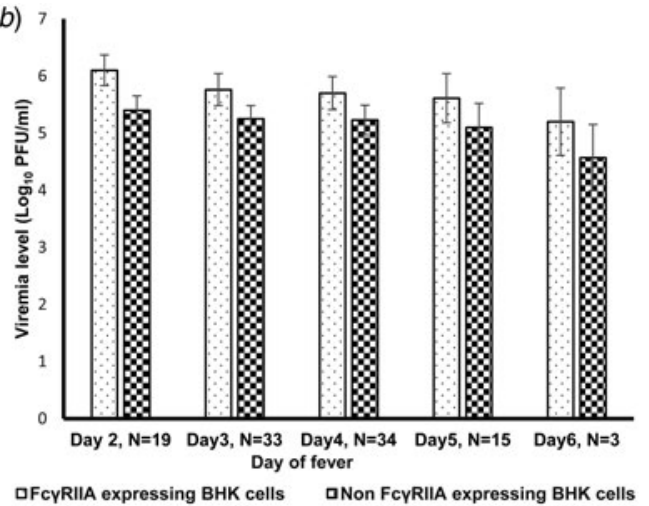

(d)

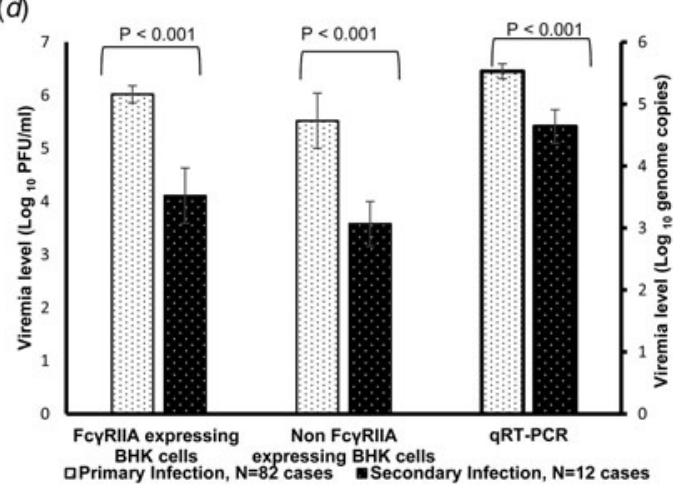

Fig. 2. Comparison of viremia levels by plaque assay (Fc $\gamma$ RIIA-expressing and non-expressing BHK cells) and/or by qRT-PCR. (a) DENV IgM-positive vs. DENV IgM-negative patients, (b) according to the day of fever of patients with isolated DENV, $(c)$ day of fever among primary infection cases, and $(d)$ primary $v s$. secondary infection. *Student's $t$ test was used for analysis.

Mandalay, 50 strains $(75.8 \%)$ of the isolated strains were DENV-1, 15 strains (22.7\%) DENV-2 and one strain $(1.5 \%)$ DENV-4. On the other hand, in Myeik, 26 strains (65\%) were DENV-1, nine strains (22.5\%) DENV-2, one strain (2.5\%) DENV-3, four strains $(10 \%)$ DENV-4. There were two instances with mixed infection of two serotypes. One instance (DENV-1 and DENV-4) was from Mandalay, Upper Myanmar and the other (DENV-1 and DENV-2) from Myeik, Lower Myanmar. 
(a)

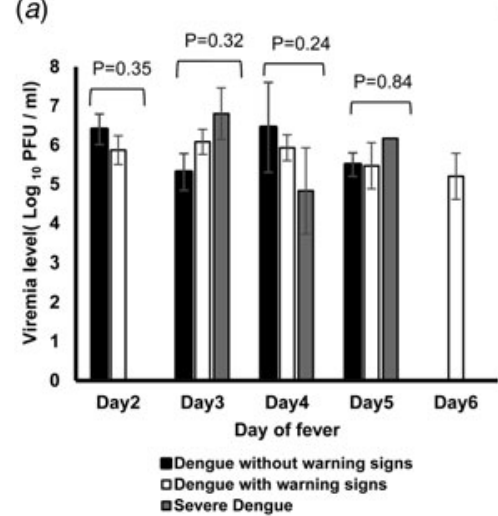

(b)

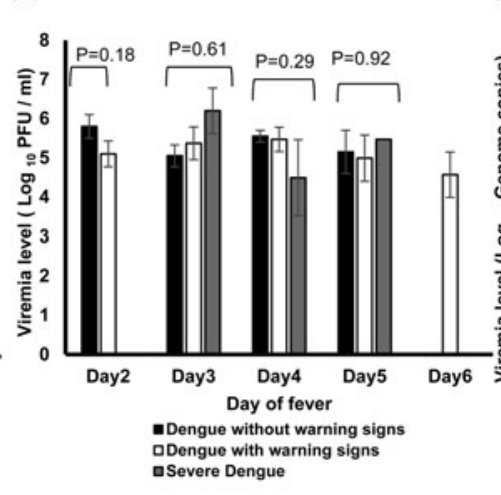

(c)

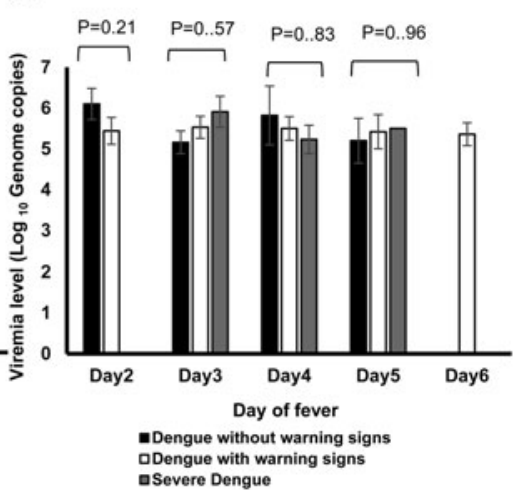

Fig. 3. Comparison of viral load levels among patients with isolated DENV and exhibiting different levels of severity of infection. (a) FcyRIIA-expressing BHK cells-based plaque assay, (b) FcyRIIA-non-expressing BHK cells-based plaque assay, and $(c)$ qRT-PCR.

Table 1. Viral loads of patients grouped according to infecting DENV serotype and disease severity

\begin{tabular}{|c|c|c|c|c|c|c|c|}
\hline \multirow{2}{*}{$\begin{array}{l}\text { Infecting serotype and disease } \\
\text { severity }\end{array}$} & \multirow{2}{*}{$\begin{array}{l}\text { Number of } \\
\text { patients } \\
N\end{array}$} & \multicolumn{2}{|c|}{$\begin{array}{l}\text { Real-time PCR } \\
\text { ( } \log _{10} \text { genome copies) }\end{array}$} & \multicolumn{4}{|c|}{ Plaque-forming Unit ( $\left.\log _{10} \mathrm{PFU} / \mathrm{ml}\right)$} \\
\hline & & & $P$ value & $\begin{array}{l}\text { Fc } \gamma \text { IIA-expressing } \\
\text { BHK cells }\end{array}$ & $P$ value & BHK cells & $P$ value \\
\hline \multicolumn{8}{|l|}{ DENV $1,2,3$, and $4 *$} \\
\hline DEN without warning signs & 27 & $5 \cdot 49 \pm 1 \cdot 11$ & $0 \cdot 93$ & $5 \cdot 73 \pm 1 \cdot 59$ & $0 \cdot 56$ & $5 \cdot 32 \pm 1 \cdot 35$ & $0 \cdot 62$ \\
\hline DEN with warning signs & 65 & $5 \cdot 48 \pm 1 \cdot 21$ & & $5 \cdot 85 \pm 1 \cdot 47$ & & $5 \cdot 26 \pm 1 \cdot 35$ & \\
\hline Severe dengue & 12 & $5 \cdot 36 \pm 0 \cdot 93$ & & $5 \cdot 33 \pm 1 \cdot 85$ & & $4 \cdot 88 \pm 1 \cdot 60$ & \\
\hline \multicolumn{8}{|l|}{ DENV-1 } \\
\hline DEN without warning signs & 17 & $5 \cdot 37 \pm 1 \cdot 12$ & $0 \cdot 93$ & $5 \cdot 62 \pm 1 \cdot 60$ & $0 \cdot 36$ & $5 \cdot 37 \pm 1 \cdot 14$ & $0 \cdot 39$ \\
\hline DEN with warning signs & 48 & $5 \cdot 47 \pm 1 \cdot 26$ & & $5 \cdot 92 \pm 1 \cdot 42$ & & $5 \cdot 39 \pm 1 \cdot 31$ & \\
\hline Severe dengue & 9 & $5 \cdot 33 \pm 1 \cdot 05$ & & $5 \cdot 14 \pm 2 \cdot 06$ & & $4 \cdot 70 \pm 1 \cdot 78$ & \\
\hline \multicolumn{8}{|l|}{ DENV-2 } \\
\hline DEN without warning signs & 8 & $5 \cdot 71 \pm 1 \cdot 21$ & $0 \cdot 92$ & $5 \cdot 90 \pm 1 \cdot 83$ & $0 \cdot 99$ & $5 \cdot 16 \pm 1 \cdot 50$ & $0 \cdot 93$ \\
\hline DEN with warning signs & 12 & $5 \cdot 41 \pm 1 \cdot 11$ & & $5 \cdot 90 \pm 1 \cdot 56$ & & $5 \cdot 08 \pm 1 \cdot 46$ & \\
\hline Severe dengue & 3 & $5 \cdot 42 \pm 0 \cdot 48$ & & $5 \cdot 87 \pm 1 \cdot 14$ & & $5 \cdot 40 \pm 0 \cdot 91$ & \\
\hline \multicolumn{8}{|l|}{ DENV-4 } \\
\hline DEN without warning signs & 1 & $6 \cdot 16 \pm 0 \cdot 00$ & $0 \cdot 88$ & $6 \cdot 70 \pm 0 \cdot 00$ & $0 \cdot 48$ & $5 \cdot 60 \pm 0 \cdot 00$ & $0 \cdot 16$ \\
\hline DEN with warning signs & 3 & $5 \cdot 98 \pm 1 \cdot 00$ & & $5 \cdot 33 \pm 1 \cdot 31$ & & $4 \cdot 73 \pm 0 \cdot 35$ & \\
\hline
\end{tabular}

Viremia level in mean \pm s.D., one-way ANOVA test was used.

* Patients were grouped together according to the levels of disease severity regardless of the infecting serotype.

\section{Phylogenetic analysis}

The phylogenetic tree based on the full coding region of E protein of DENV-1 strains from both study areas in Myanmar shows that all the strains belonged to genotype 1 (Fig. 4). The 50 strains from Upper Myanmar were subclustered into three distinct lineages but the 26 strains from Lower Myanmar fell within one lineage. Most strains had 99\% nucleotide similarity to the previous strains circulating in Myanmar. The isolated strains were closely related from the strains circulating in China, Thailand, Sri Lanka and the strains previously isolated in Myanmar. Moreover, there was no association between the disease severity and specific clade of DENV-1 based on the phylogenetic analysis.

All the isolated DENV-2 from the two study areas fell under Asian I genotype and formed two lineages. Most of the strains were closely similar to the strains circulating in Thailand, China, and Myanmar strains and had $99 \%$ nucleotide similarity to the previously 


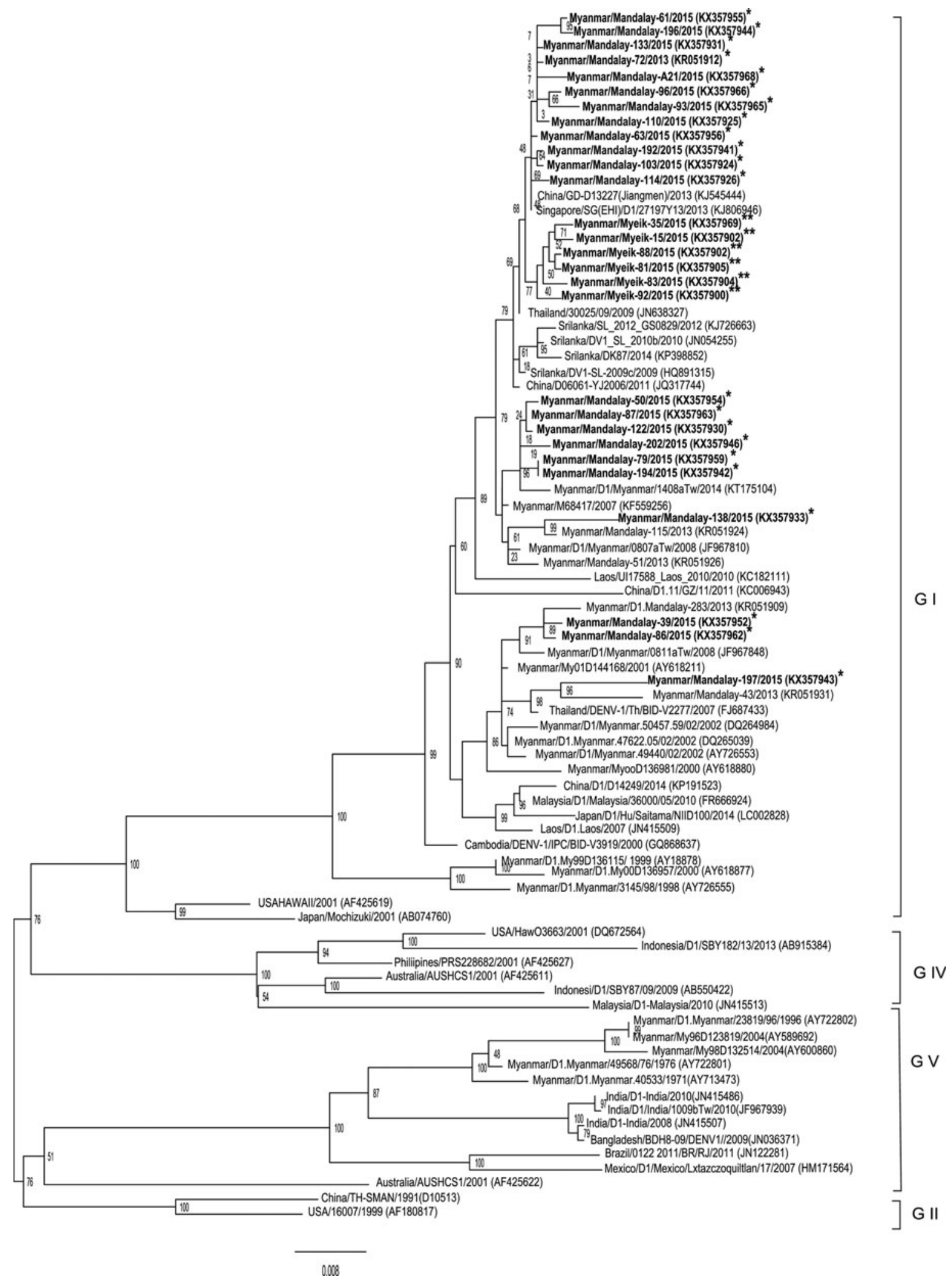

Fig. 4. DENV-1 phylogenetic tree. Phylogenetic tree was constructed based on the whole nucleotide sequences of the E protein gene of DENV-1 showing the relationship of 82 strains from different sources including 28 strains of DENV-1 isolated during the 2015 epidemic in Myanmar. The representative strains of each genotype obtained from Genbank are named by country origin, strain name, year of isolation and GenBank accession number. *Upper Myanmar; **Lower Myanmar. 


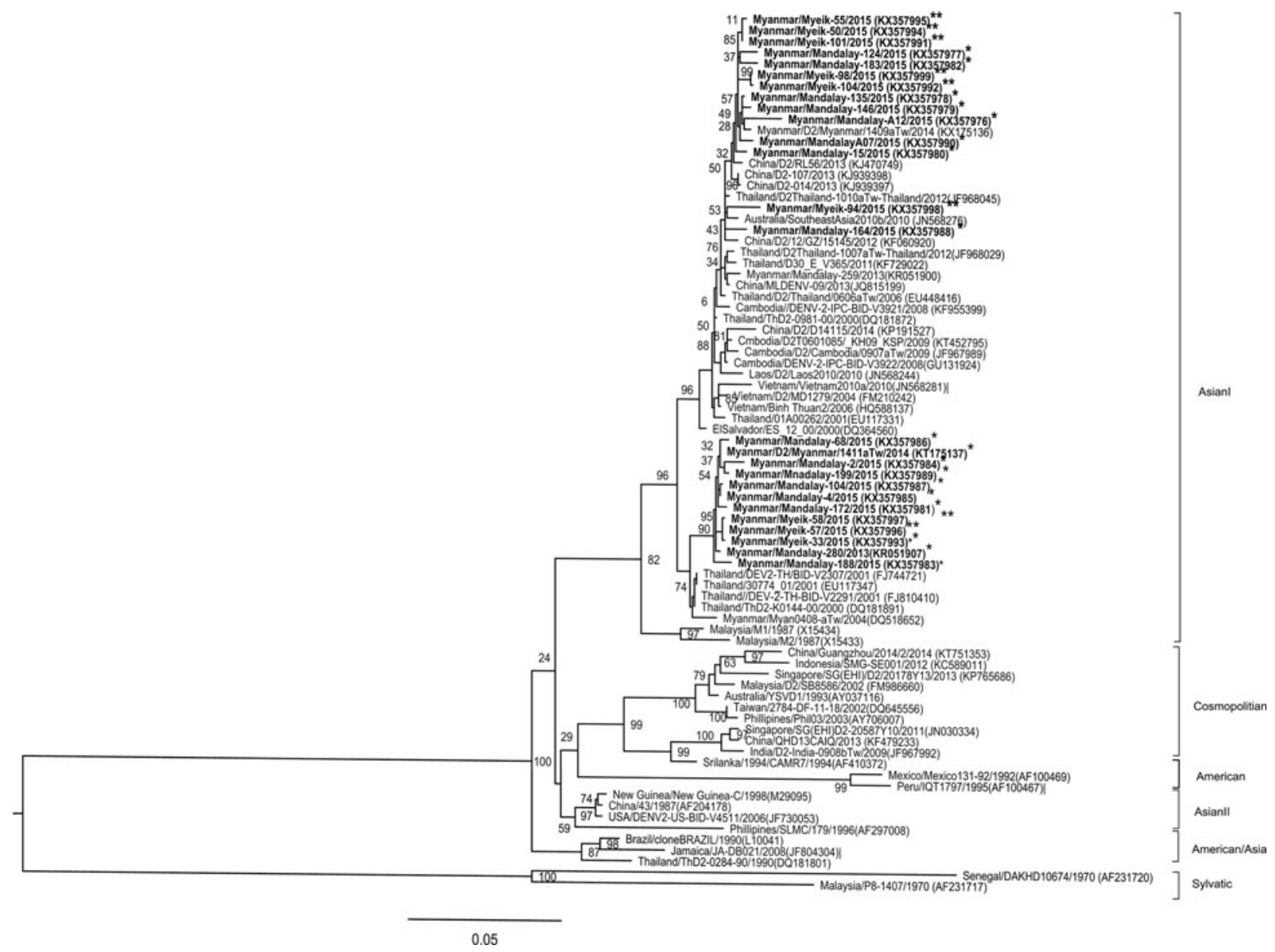

Fig. 5. DENV-2 phylogenetic tree. Phylogenetic tree was constructed based on the whole nucleotide sequences of the E protein gene of DENV-2 showing the relationship of 79 strains from different sources including 24 strains of DENV-2 isolated during the 2015 epidemic in Myanmar. The representative strains of each genotype obtained from Genbank are named by country origin, strain name, year of isolation and GenBank accession number. *Upper Myanmar; **Lower Myanmar.

isolated strains in Myanmar (Fig. 5). The only DENV-3 isolate from this study and which came from Lower Myanmar belonged to the genotype III. This strain was closely related to the strains circulating in Thailand, Laos, Cambodia, and Vietnam (Supplementary Fig. S2). The four DENV-4 strains from Lower Myanmar and the one strain from Upper Myanmar belonged to genotype I (Supplementary Fig. S3). The strains were similar to the strains from Myanmar and Thailand.

\section{DISCUSSION}

In Myanmar, the regular incidence of hospitalized DEN patients is about 7000-9000 cases per year and can reach more than 15000 per year during an outbreak according to the hospital statistics of the Ministry of Health and Sports. During the DEN epidemics in 2009, 2013, and 2015, the incidence rates were 24285,20255 , and 42913 cases, respectively. The total reported cases for 2015 was comparable to the total cases during the last 5 years from 2010 to 2014 indicating that the 2015 outbreak was the biggest to this date. Although the incidence rate in 2015 was very high, the mortality rate $(0 \cdot 32 \%)$ decreased in numbers compared with the previous years. This reduction in mortality rate could be due to early diagnosis, effective treatment and timely referral system in Myanmar [7] which were made possible through updating of the management guidelines for epidemic preparedness and response and treatment for dengue. In addition are the capacity building for the medical officers for the early recognition of early warning signs and the political commitment of the government for the technical and material support (source: Ministry of Health and Sports, Myanmar). 
In the present study, DENV-1 was the most prevalent serotype in the two study sites in Myanmar during the 2015 outbreak. Although the samples were collected from two study areas with different geographical and environmental background, the DENV serotype and genotype distribution pattern was similar in the two regions. The DENV circulating during this outbreak were heterogeneous having similarities not only from the strains previously isolated in Myanmar but also to the strains from neighboring countries. Not only virus factor but also demographic data (age, sex) of laboratory-confirmed DEN patients in this study was similar to the results of previous surveillance data in Myanmar [7, 8]. For clinical manifestation, there were no specific clinical signs and symptoms associated with each serotype of DENV. The number of patients with specific serotypes were limited, hence it was difficult to make conclusion about the association of clinical presentation with respect to serotype.

Haematocrit values differed among the three groups of patients with different disease severity and were highest in patients with severe dengue due to plasma leakage. Platelet count was lowest in patients with severe dengue, followed by those with warning signs. The reduced platelet count could be due to bone marrow suppression of platelet production or increased destruction. According to WHO guidelines, these two laboratory markers are important parameters for predicting severe dengue [1] which were confirmed in this study.

A noticeably high number of primary infection was noted among severe DEN cases in this study. To this date, the prevalence of severe DEN during primary infection has been high in Myanmar. Some South East Asian countries (Philippines, Indonesia, and Thailand,) also reported the high percentage of severe dengue with primary infection [7]. Many factors are involved in the pathogenesis of severe dengue with primary infection. Individual host factors (genetic background, underlying diseases, nutritional status, immune response) could be involved in the pathogenesis of severe DEN with primary infection [8]. Among 12 severe dengue patients with primary infection in this study, six patients $(50 \%)$ were infected with DENV-1 and three patients with DENV-2, however the infecting serotype of the last three patients were unknown due to the failure of virus isolation. The virulence factors of the circulating DENV (genotype and phenotype) could be involved in the pathogenesis of severe dengue [8]. The study from Singapore found that DENV-1 (genotype 1) and DENV-2
(Cosmopolitan genotype) can cause severe dengue than other serotypes or genotypes [23]. Similarly, DENV-1 (Genotype 1) and DENV-2 were dominant in this outbreak but DENV-2 strains were of the Asian-1 genotype. Furthermore, some mutant virus strains could change to virulent form and could cause severe disease without antibody dependent enhancement (ADE) phenomenon [24].

It was also observed in this study that the viremia level was significantly higher among patients with primary infection (Fig. 2d). One study reported that the peak viremia level was up to 3 days and gradually decreased $2 \cdot 2 \log _{10}$ per day [25]. In this study, the viral loads were consistently high during primary infection up to day 5 of fever (Fig. 2c), but no significant difference was observed in the viral loads among the three different severity groups. Analysis done based on or regardless of the infecting serotype showed no differences between these groups (Table 1). Therefore, not only high viremia level but also many factors could be involved in the high rate of severe dengue with primary infection in Myanmar.

In this study, viremia levels were also higher among patients negative for IgM antibody compared with the positive ones $(P$ value $<0 \cdot 05)$. The presence of DENV-specific IgM antibody could influence the viremia level of the patients in this study. Based on the literature review, the presence of specific IgM antibody helps clear the virions through their uptake by phagocytes [26]. Moreover, one study also proved that the viremia level decreased when the IgM antibody appeared in blood and this made the isolation of the virus difficult [27].

Generally, qRT-PCR results expressed the number of biological molecules, i.e. the copy number of virus genome but not the infectious potential of the virus. To determine the biological infectious properties, plaque assays should be done [28]. Results of qRT-PCR could not be equated with the results of plaque assays because the presence of defective non-infectious particles detected by qRT-PCR could not be detected by plaque assays and thus the results could be misleading [29]. There were previous studies on the comparison of viremia level with the different degrees of clinical severity based only on qRT-PCR method [30, 31]. Therefore, this study included the plaque assay method in addition to qRT-PCR method to assess the correlation of viremia and disease severity. In this study, there were comparable results between these two diagnostic methods. The samples collected in this study were fresh and there was no repeated 
freeze and thawing procedures that occurred. The viremia level of $\mathrm{Fcy}$-expressing cells were higher in this study because the presence of $\mathrm{Fc} \gamma$ receptor plays an important role in DENV pathogenesis such as virus initiation and replication stage of DENV infection [32].

The high plasma viremia level of DEN patients is an important marker to be able to spread DENV from infected human to mosquitoes during an outbreak. The viremia level above the mosquito infectious dose $\left(\mathrm{MID}_{50}\right)$ could play as a source of infection for spreading the disease [33]. Most DENV-1 and DENV-2 patients from our study had high viremia level above the MID $_{50}$ when compared with the study conducted in Vietnam [33]. A. aegypti mosquitoes feeding on viremic patients can spread the virus to another person after at least 11 days of extrinsic incubation [34]. In our study, the patients with high viremia level (above $\mathrm{MID}_{50}$ ) was observed up to day 6 of fever. Thus, the presence of dengue patients that were highly viremic for a long span of time could be the source of sustaining the 2015 large epidemic in Myanmar.

According to the phylogenetic tree analysis based on the full coding region of the E protein, the genotypes of the circulating DENV serotypes in both study areas were not different. All DENV-1 isolates were genotype I with three distinct clades but the viral strains from Myeik subclustered to one unique clade. The DENV-1 strains from Mandalay in 2013 belonged to Genotype 1 and were distributed into three distinct clades [7]. The DENV-1 isolates in 2015 belonged to the same three clades as in 2013 .

Although previous studies reported that both Asian I and Cosmopolitan genotypes of DENV-2 were circulating in Myanmar, only Asian I genotype with two distinct clades was isolated in the 2013 [7] and 2015 outbreaks. Similarly in DENV-3, two genotypes (II and III) were previously circulating in Myanmar [35] but only Genotype III was isolated in 2015. For DENV-4, only Genotype I was found circulating in Myanmar. These studies proved that different DENV serotypes and genotypes (except for DENV-4) have been co-circulating in Myanmar [7].

In conclusion, all four DENV serotypes were confirmed to be concurrently circulating and causing epidemic in Myanmar with DENV-1 as the most dominant serotype. Additionally, the number of severe DEN patients with primary infection were still high during the 2015 outbreak. Patients with primary infection demonstrated high level of viremia but there was no association between the viral loads and disease severity. The high viral load with a long duration among the viremic DEN patients could perhaps serve as the increased source of infection to support the transmission of DENV through the vector mosquitoes during this epidemic.

\section{SUPPLEMENTARY MATERIAL}

The supplementary material for this article can be found at https://doi.org/10.1017/S0950268817000735

\section{ACKNOWLEDGEMENTS}

The authors would like to thank all the patients who participated in this study. They are also grateful to all the members of the Department of Virology, Institute of Tropical Medicine, Nagasaki University and of the Virology Research Division, Department of Medical Research (Pyin Oo Lwin Branch). A.K. Kyaw is a recipient of a MEXT PhD scholarship in Japan. This work was supported financially by a Health and Labour Sciences Research Grant on Emerging and Re-emerging Infectious Diseases from the Japanese Ministry of Health, Labor and Welfare, Health and Labor Sciences Research Grants (Grants in aid for AMED, H26- shinkou-jitsuyouka-007). This research was partially supported by the Japan Initiative for Global Research Network on Infectious Diseases; Japan-US Cooperative Medical Program from Japan Agency for Medical Research and Development (AMED); and Joint Usage/Research Center on Tropical Disease, Institute of Tropical Medicine, Nagasaki University. The funders had no role in study design, data collection and analysis, decision to publish, or preparation of the manuscript.

\section{DECLARATION OF INTEREST}

None.

\section{REFERENCES}

1. Anon. Dengue: Guidelines for Diagnosis, Treatment, Prevention and Control, New Edition. Geneva: World Health Organization, 2009.

2. Bhatt $\mathbf{S}$, et al. The global distribution and burden of dengue. Nature 2013; 496(7446): 504-507.

3. Shepard DS, Undurraga EA, Halasa YA. Economic and disease burden of dengue in Southeast Asia. PloS Neglected Tropical Diseases 2013; 7(2): e2055. 
4. Struchiner CJ, et al. Increasing dengue incidence in Singapore over the past 40 years: population growth, climate and mobility. PLoS ONE 2015; 10(8): e0136286.

5. Thu HM, et al. Myanmar dengue outbreak associated with displacement of serotypes 2,3 , and 4 by dengue 1. Emerging Infectious Diseases 2004; 10(4): 593-597.

6. Myat Thu $\mathbf{H}$, et al. Lineage extinction and replacement in dengue type 1 virus populations are due to stochastic events rather than to natural selection. Virology 2005; 336(2): 163-172.

7. Ngwe Tun MM, et al. Characterization of the 2013 dengue epidemic in Myanmar with dengue virus 1 as the dominant serotype. Infection, Genetics and Evolution 2016; 43: 31-37.

8. Ngwe Tun MM, et al. Serological characterization of dengue virus infections observed among dengue hemorrhagic fever/dengue shock syndrome cases in upper Myanmar. Journal of Medical Virology 2013; 85(7): $1258-1266$.

9. Khai Ming C, et al. Clinical and laboratory studies on haemorrhagic fever in Burma, 1970-72. Bulletin of the World Health Organization 1974; 51(3): 227-235.

10. Murray NE, Quam MB, Wilder-Smith A. Epidemiology of dengue: past, present and future prospects. Journal of Clinical Epidemiology 2013; 5: 299-309.

11. Vaughn DW, et al. Dengue viremia titer, antibody response pattern, and virus serotype correlate with disease severity. The Journal of Infectious Diseases 2000; 181(1): 2-9.

12. Wang WK, et al. High levels of plasma dengue viral load during defervescence in patients with dengue hemorrhagic fever: implications for pathogenesis. Virology 2003; 305(2): 330-338.

13. Wang WK, et al. Slower rates of clearance of viral load and virus-containing immune complexes in patients with dengue hemorrhagic fever. Clinical Infectious Diseases 2006; 43(8): 1023-1030.

14. Gubler DJ, et al. Epidemic dengue hemorrhagic fever in rural Indonesia. I. Virological and epidemiological studies. The American Journal of Tropical Medicine and Hygiene 1979; 28(4): 701-710.

15. Kuberski T, et al. Clinical and laboratory observations on patients with primary and secondary dengue type 1 infections with hemorrhagic manifestations in Fiji. The American Journal of Tropical Medicine and Hygiene 1977; 26(4): 775-783.

16. Pal S, et al. Evaluation of dengue NS1 antigen rapid tests and ELISA kits using clinical samples. PLoS ONE 2014; 9(11): e113411.

17. Inoue S, et al. Evaluation of a dengue $\mathrm{IgG}$ indirect enzyme-linked immunosorbent assay and a Japanese encephalitis IgG indirect enzyme-linked immunosorbent assay for diagnosis of secondary dengue virus infection. Vector-Borne and Zoonotic Diseases 2010; 10(2): 143-150.

18. Moi ML, et al. Detection of higher levels of dengue viremia using FcyR-expressing BHK-21 cells than Fc $\gamma$ R-negative cells in secondary infection but not in primary infection. The Journal of Infectious Diseases 2011; 203(10): 1405-1414.
19. Igarashi A. Isolation of a Singh's Aedes albopictus cell clone sensitive to Dengue and Chikungunya viruses. Journal of General Virology 1978; 40(3): 531-544.

20. Lanciotti RS, et al. Rapid detection and typing of dengue viruses from clinical samples by using reverse transcriptase-polymerase chain reaction. Journal of Clinical Microbiology 1992; 30(3): 545-551.

21. Morita K, Tanaka M, Igarashi A. Rapid identification of dengue virus serotypes by using polymerase chain reaction. Journal of Clinical Microbiology 1991; 29 (10): 2107-2110.

22. Guindon S, et al. New algorithms and methods to estimate maximum-likelihood phylogenies: assessing the performance of PhyML 3.0. Systematic Biology England 2010; 59(3): 307-321.

23. Yung CF, et al. Dengue serotype-specific differences in clinical manifestation, laboratory parameters and risk of severe disease in adults, Singapore. The American Journal of Tropical Medicine and Hygiene 2015; 92(5): 999-1005.

24. Pongsumpun P, Yoksan S, Tan IM. A comparison of the age distributions in the dengue hemorrhagic fever epidemics in Santiago de Cuba (1997) and Thailand (1998). The Southeast Asian Journal of Tropical Medicine and Public Health 2002; 33(2): 255-258.

25. Tricou V, et al. Kinetics of viremia and NS1 antigenemia are shaped by immune status and virus serotype in adults with dengue. PLoS Neglected Tropical Diseases 2011; 5(9): e1309.

26. Ehrenstein MR, Notley CA. The importance of natural IgM: scavenger, protector and regulator. Nature Reviews Immunology 2010; 10(11): 778-786.

27. Jarman RG, et al. Factors influencing dengue virus isolation by $\mathrm{C} 6 / 36$ cell culture and mosquito inoculation of nested PCR-positive clinical samples. The American Journal of Tropical Medicine and Hygiene 2011; 84(2): 218-223.

28. Bae HG, et al. Detection of yellow fever virus: a comparison of quantitative real-time PCR and plaque assay. Journal of Virological Methods 2003; 110(2): 185191.

29. Morozov VA, Weiss RA. Two types of HTLV-1 particles are released from MT-2 cells. Virology 1999; 255(2): 279-284.

30. Thai KT, et al. Clinical, epidemiological and virological features of dengue virus infections in Vietnamese patients presenting to primary care facilities with acute undifferentiated fever. Journal of Infection 2010; 60(3): 229-237.

31. Libraty DH, et al. Differing influences of virus burden and immune activation on disease severity in secondary dengue-3 virus infections. The Journal of Infectious Diseases 2002; 185(9): 1213-1221.

32. Moi ML, et al. Development of an antibodydependent enhancement assay for dengue virus using stable BHK-21 cell lines expressing Fc gammaRIIA. Journal of Virological Methods 2010; 163(2): 205-209.

33. Nguyet MN, et al. Host and viral features of human dengue cases shape the population of infected and 
infectious Aedes aegypti mosquitoes. Proceedings of the National Academy of Sciences USA 2013; 110(22): 9072-9077.

34. Nishiura H, Halstead SB. Natural history of dengue virus (DENV)-1 and DENV-4 infections: reanalysis of classic studies. The Journal of Infectious Diseases 2007; 195(7): 1007-1013.

35. Thant KZ, et al. Molecular epidemiology of dengue viruses co-circulating in upper Myanmar in 2006. Tropical Medicine and Health 2015; 43(1): 21-27. 